$02,05,13$

\title{
Радиационная обработка краев трещины сверхпроводящего слоя при реставрации ВТСП-ленты
}

\author{
(С) А.И. Подливаев ${ }^{1,2}$, И.А. Руднев ${ }^{1}$ \\ ${ }^{1}$ Национальный исследовательский ядерный университет „МИФИ“, \\ Москва, Россия \\ ${ }^{2}$ Научно-исследовательский институт проблем развития научно-образовательного потенциала молодежи, \\ Москва, Россия \\ E-mail: AlPodlivayev@mephi.ru
}

Поступила в Редакцию 5 ноября 2021 г.

В окончательной редакции 8 ноября 2021 г.

Принята к публикации 9 ноября 2021 г.

\begin{abstract}
Численно, в рамках модели критического состояния определена плотность сверхпроводящих токов в ВТСП-ленте второго поколения на основе $\mathrm{GdBa}_{2} \mathrm{Cu}_{3} \mathrm{O}_{7-x}$. Показано, что при реставрации поперечной трещины сверхпроводящего слоя путем шунтирования трещины отрезком бездефектной ленты, критический ток реставрированного участка уменьшается на $\sim 8 \%$. Показано, что предварительное радиационное облучение краев трещины ионами водорода, гелия, неона и кислорода позволяет восстановить первоначальное значение критического тока. Расчет воздействия излучения на сверхпроводящую ленту проводился с использованием комплекса программ SRIM.
\end{abstract}

Ключевые слова: высокотемпературный сверхпроводник, облучение, радиационные дефекты, критический ток.

DOI: $10.21883 /$ FTT.2022.03.52092.233

\section{1. Введение}

Современные высокотемпературные сверхпроводящие (ВТСП) композитные ленты второго поколения на основе соединений $\mathrm{YBa}_{2} \mathrm{Cu}_{3} \mathrm{O}_{7-x}$ или $\mathrm{GdBaCuO}$ обладают высокими значениями критического тока, благодаря чему широко используются в области электроэнергетики, при создании высокоэффективных линий электропередач, моторов, генераторов, ограничителей тока и т. п. ВТСП-композиты также обладают высокими значениями плотности критического тока в сильных магнитных полях, что делает их крайне перспективным материалом для использования в сверхпроводящих магнитных установках класса МЕГА-сайнс (большой адронный коллайдер БАК, ускоритель НИКА, проект ИТЭР и др.). В этих установках ВТСП находятся в условиях сильных радиационных полей, неизбежно приводящих к изменению сверхпроводящих характеристик, прежде всего критического тока и критической температуры, из-за образования в ВТСП-материале радиационных дефектов. В зависимости от типа сверхпроводника и характера облучения радиационное воздействие может существенно влиять на локальную плотность критического тока, при этом зависимость этой величины от дозы может быть немонотонной. При малой дозе в некоторых случаях наблюдается рост, при большой - падение плотности критического тока. Так, например, подобная немонотонность представлена в работе [1], где сверхпроводящая пленка $\mathrm{GdBaCuO}$ облучалась ионами $\mathrm{Zr}$, работе [2], где пленки ВТСП подвергались нейтронному облучению или в [3], где сверхпроводящая пленка $\mathrm{GdBaCuO}$, покрытая слоем серебра, облучалась ионами Ar, Хе и $\mathrm{Kr}$. Повышение плотности критического тока связано с микроскопическими неоднородностями распределения радиационных дефектов, являющимися центрами пиннинга для вихрей Абрикосова в сверхпроводнике. Понижение плотности критического тока при облучении связано с разупорядочением кристаллической структуры сверхпроводника.

Неоднородность распределения дефектов в сверхпроводнике может приводить также к локальной неоднородности изменения сверхпроводящих характеристик в объеме материала. В свою очередь, такая неоднородность может сопровождаться необычными физическими явлениями, например, появлением фазовых переходов по току, аналогичных наблюдаемым в гранулированных сверхпроводниках $\mathrm{YBa}_{2} \mathrm{Cu}_{3} \mathrm{O}_{7-x}$ [4].

$\mathrm{B}$ настоящей работе показана парадоксальная ситуация, когда радиационное понижение плотности критического тока в макроскопической области дефектной ВТСП-ленты может приводить к возрастанию полного критического транспортного тока через эту сверхпроводящую систему.

В качестве дефекта ВТСП-ленты изучается трещина сверхпроводящего слоя. Существенным недостатком ВТСП $\mathrm{YBa}_{2} \mathrm{Cu}_{3} \mathrm{O}_{7-x}$ и $\mathrm{GdBa}_{2} \mathrm{Cu}_{3} \mathrm{O}_{7-x}$ является хрупкость этих материалов. Чрезмерный изгиб сверхпроводящей ленты приводит к возникновению поперечных трещин в ленте, что делает ее непригодной к использованию в транспортных токовых системах (см., например [5]). Простейшим способом восстановления 


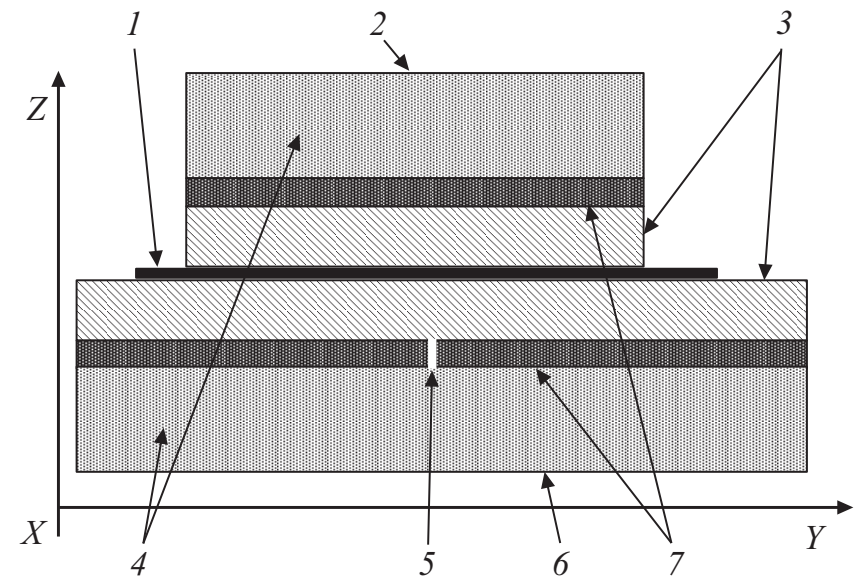

Рис. 1. ВТСП-лента с поперечной трещиной и припаянный к ней шунт (вид сбоку). 1 - припой, обеспечивающий электрический контакт дефектной ленты и шунта. 2 - шунт, 3 - защитное покрытие ВТСП-ленты и шунта, 4 - подложки ленты и шунта, 5 - трещина ВТСП-слоя, 6 - ВТСП-лента с трещиной, 7 - сверхпроводящие слои ленты и шунта.

токонесущих характеристик сверхпроводящей линии на основе отдельной ВТСП-ленты является шунтирование участка с поперечной трещиной отрезком аналогичной бездефектной ленты, имеющим хороший электрический контакт с поврежденной лентой в окрестности трещины. Пайка ВТСП-лент является обычным приемом, который обеспечивает хороший электрический контакт при их соединении (см., например [6]).

На рис. 1 представлен вид сбоку композитной ВТСПленты с поперечной трещиной, которая закорочена отрезком бездефектной ленты аналогичного типа. Также на этом рисунке определена координирующая ленту декартова система $(X, Y, Z)$. В настоящей работе изучается распределение плотности сверхпроводящих токов в данной структуре в случаях, когда область вблизи трещины не подвергалась предварительной радиационному облучению, и когда эта область облучалась быстрыми ионами до присоединения к ленте шунта.

Статья имеет следующую структуру: в разделе 2 в рамках модели критического состояния определяется плотность сверхпроводящих токов в шунтированной ВТСП-ленте с трещиной без предварительной радиационной обработки поврежденного участка. В разделе 3 для определенного неоднородного профиля радиационных дефектов, возникающих при ионном облучении, также определяется плотность сверхпроводящих токов в изучаемой системе. В начале этого раздела приводится расчет режимов облучения, необходимых для образования концентрации радиационных дефектов желаемого профиля. Расчет радиационного повреждения ВТСП-ленты проводится с привлечением программного пакета SRIM (Stopping and Range of Ions in Matter [7]).

\section{2. Сверхпроводящие токи в отсутствие радиационной обработки}

\section{1. Постановка задачи}

Определение индуцированных сверхпроводящих токов в верхнем сверхпроводящем слое (ВСС), наведенных полем магнитной линейки возможно в рамках различных подходов. Для описания критических токов в тонкой сверхпроводящей пленке можно использовать подход, основанный на решении уравнений ГинзбургаЛандау [8]. Этот подход позволяет детально определить структуру сверхпроводящих токов, включая структуру отдельного сверхпроводящего вихря, но при решении макроскопических задач требует слишком высоких затрат компьютерных ресурсов. Более упрощенный подход при описании системы токов оперирует с системой абрикосовских вихрей в магнитном поле, где вихри рассматриваются как отдельные, взаимодействующие друг с другом частицы (смотри, например, [9]), однако и данный подход требует чрезмерных затрат компьютерных ресурсов.

Изучаемым объектом, также, как и в работах [10-12] является ВТСП-лента $\mathrm{GdBa}_{2} \mathrm{Cu}_{3} \mathrm{O}_{7-x}$ производства фирмы СуперОкс. В отсутствие внешнего магнитного поля величина объемной плотности критического тока при температуре жидкого азота $J_{c}=2.77 \cdot 10^{6} \mathrm{~A} / \mathrm{cm}^{2}$ [13]. Ширина ленты $a$ равна $12 \mathrm{~mm}(-a / 2<X<a / 2)$. Толщина ВТСП-слоя $d$ (направление оси $Z$ ) в ленте также, как и в работах [10-12] равна $1.5 \mu \mathrm{m}$. Связь объемной плотности критического тока $J_{c}$ и плотности двумерного критического тока $j_{c}$ ВТСП ленты определяется следующим соотношением $j_{c}=J_{c} \cdot d$. В соответствие с данными работы [13] защитное покрытие ленты состоит из прилежащего к сверхпроводнику слоя серебра толщиной $2 \mu \mathrm{m}$ и покрывающего его слоя меди толщиной $20 \mu \mathrm{m}$. Подложка из сплава Hastelloy C-276 $(\mathrm{Ni}, \mathrm{Mo}, \mathrm{Cr}, \mathrm{Fe}, \mathrm{W})$ изолирована от сверхпроводника тонким слоем оксидов металлов. При необходимости электрическое сопротивление защитного покрытия $(\mathrm{Cu}$, $\mathrm{Ag}$ ) между лентой и шунтом может быть уменьшено удалением медного слоя, и в настоящей работе этим сопротивлением мы пренебрегаем. В рамках этих предположений и двухэкспоненциального приближения зависимости критического тока от величины индукции магнитного поля В [14] плотность двумерного критического тока $j_{c}$ системы шунт-ВТСП лента с трещиной может быть представлена в следующем виде:

$$
\begin{gathered}
j_{c}(B, Y)=C(B) \cdot h(Y), \\
C(B)=\left(A_{1} \cdot \exp \left(-|B| / \beta_{1}\right)+\left(A_{2} \cdot \exp \left(-|B| / \beta_{2}\right)\right) .\right.
\end{gathered}
$$

Параметры приближения $A_{1}=23665 \mathrm{~A} / \mathrm{m}, A_{2}=$ $=17884 \mathrm{~A} / \mathrm{m}, \quad \beta_{1}=0.1175 \mathrm{~T}$ и $\beta_{2}=1.2238 \mathrm{~T}$ определяются подгонкой к экспериментальной зависимости работы [13] методом наименыших квадратов. В от- 


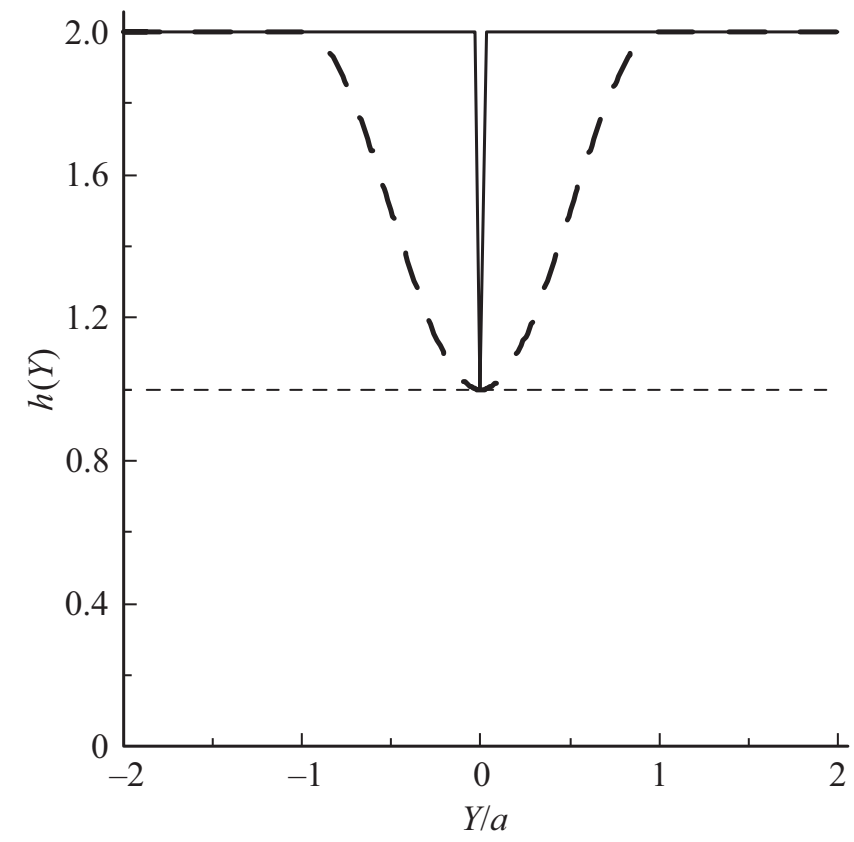

Рис. 2. Зависимость параметра $h$ от продольной координаты $Y$. Тонкая пунктирная линия - одинарная ВТСП-лента без трещины. Сплошная тонкая линия - система шунт-лента с трещиной в отсутствие предварительной радиационной обработки краев трещины. Жирная пунктирная линия - система шунт-лента с трещиной после предварительной радиационной обработки краев трещины.

дельной ленте без трещины безразмерный параметр $h=1$. Для системы шунт-ВТСП-лента с поперечной трещиной при $Y=0$ параметр $h=2$ при $Y \neq 0$ и $h=1$ при $Y=0$ (см. рис. 2).

Связь магнитного поля $\mathbf{B}(x, Y, Z, t)$ с электрическим полем $\mathbf{E}(X, Y, Z, t)$ определяется законом индукции

$$
\frac{\partial \mathbf{B}(X, Y, Z, t)}{\partial t}=-\nabla \times \mathbf{E}(X, Y, Z, t) .
$$

На двумерной структуре (плоскость $Z=0$ ):

$$
\frac{\partial B_{Z}(X, Y, 0, t)}{\partial t}=-\frac{\partial E_{X}(X, Y, 0, t)}{\partial Y}+\frac{\partial E_{Y}(X, Y, 0, t)}{\partial X} .
$$

Завершает систему уравнений нелинейный закон Ома для ВТСП-пленки

$$
\mathbf{E}(X, Y, 0, t)=\rho(\mathbf{j}(X, Y, t), B(X, Y, 0, t)) \cdot \mathbf{j}(X, Y, t) .
$$

Зависимость двумерного удельного сопротивления $\rho(\mathbf{j}, B)$ часто выбирается в виде

$$
\rho(\mathbf{j}, B)=\rho_{0}\left[|\mathbf{j}| / j_{c}(B)\right]^{n}, \quad \mathbf{j}=\sqrt{j_{X}^{2}(X, Y, t)+j_{Y}^{2}(X, Y, t)}
$$

(см., например $[15,16])$, где обычно $n \sim 20-50$. В настоящей работе, так же как в $[10-12,17,18]$, мы выбираем зависимость $\rho(\mathbf{j}, B)$ следующим образом:

$$
\rho(\mathbf{j}, B)= \begin{cases}0, & |\mathbf{j}|<j_{c}(B) \\ \rho_{0} \cdot\left[\mathbf{j} \mid-j_{c}(B)\right]^{2}, & j_{c}(B) \leq|\mathbf{j}|,\end{cases}
$$

Детальное описание алгоритма численного решения данной задачи представлено в работах $[17,18]$, и, ввиду громоздкости, в данной статье не приводится. Представленная модель адекватно (соответствуя экспериментальным данным) описывала сверхпроводящие характеристики пластин ВТСП различной формы.

\section{2. Результаты и обсуждение}

В отсутствие зависимости плотности критического тока от магнитного поля при $j_{c}=A_{1}+A_{2}=41549 \mathrm{~A} / \mathrm{m}$ (см. выражение (1)), величина критического транспортного тока через одинарную бездефектную ВТСП-ленту $I_{\mathrm{c}}=500 \mathrm{~A}$. Учет полевой зависимости критического тока от магнитного поля при решении задачи $(1-5)$ для одинарной бездефектной ВТСП-ленты снижает эту величину до значения $I_{\mathrm{c}}=473 \mathrm{~A}$. Плотность тока и наведенное им магнитное поле в бездефектной ВТСП-ленте не изменяется в продольном направлении (ось $Y$ ). На рис. 3 представлены распределения критической плотности сверхпроводящего тока и соответствующего магнитного поля в поперечном (ось $X$ ) направлении. На этом рисунке видно, что плотность критического тока максимальна в центре ленты, где магнитное поле равно нулю и монотонно понижается по мере приближения к краям, вблизи которых магнитное поле сравнительно велико и подавляет сверхпроводимость.

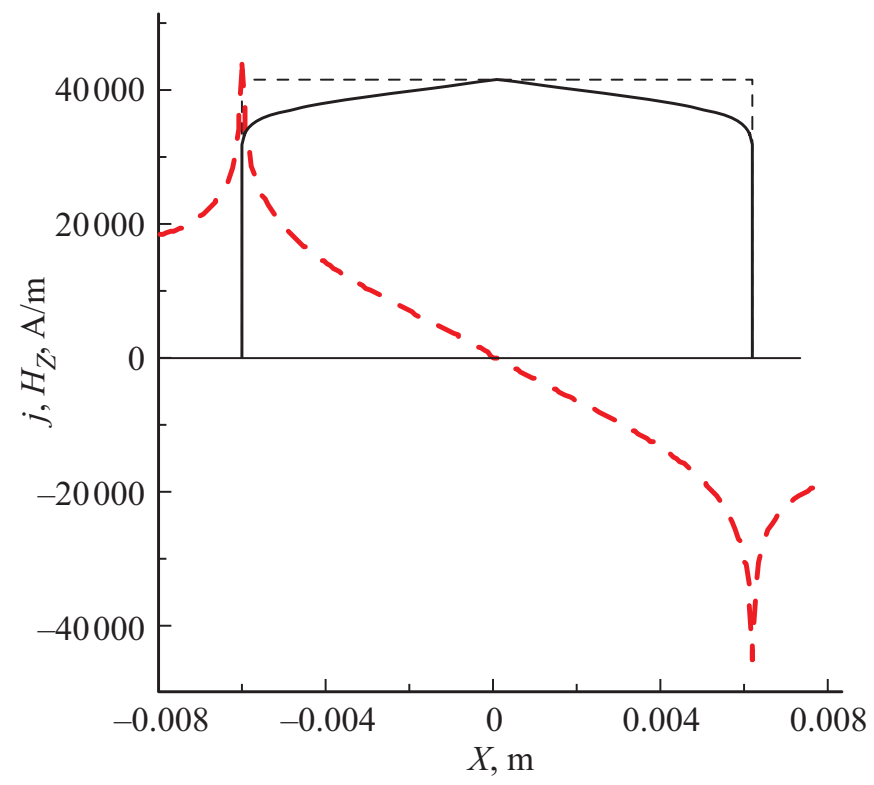

Рис. 3. Зависимость плотности сверхпроводящего тока и амплитуды наведенного им магнитного поля в однослойной бездефектной ВТСП-ленте. Компонента магнитного поля, нормальная к поверхности ленты $H_{Z}=B_{Z} / \mu_{0}, \mu_{0}$ - магнитная постоянная. Тонкая черная пунктирная линия - значение критического тока при нулевом магнитном поле. Тонкая черная сплошная линия - значение критического тока при учете магнитополевой зависимости в двухэкспоненциальном приближении (2). Жирная красная пунктирная линия - амплитуда магнитного поля. 


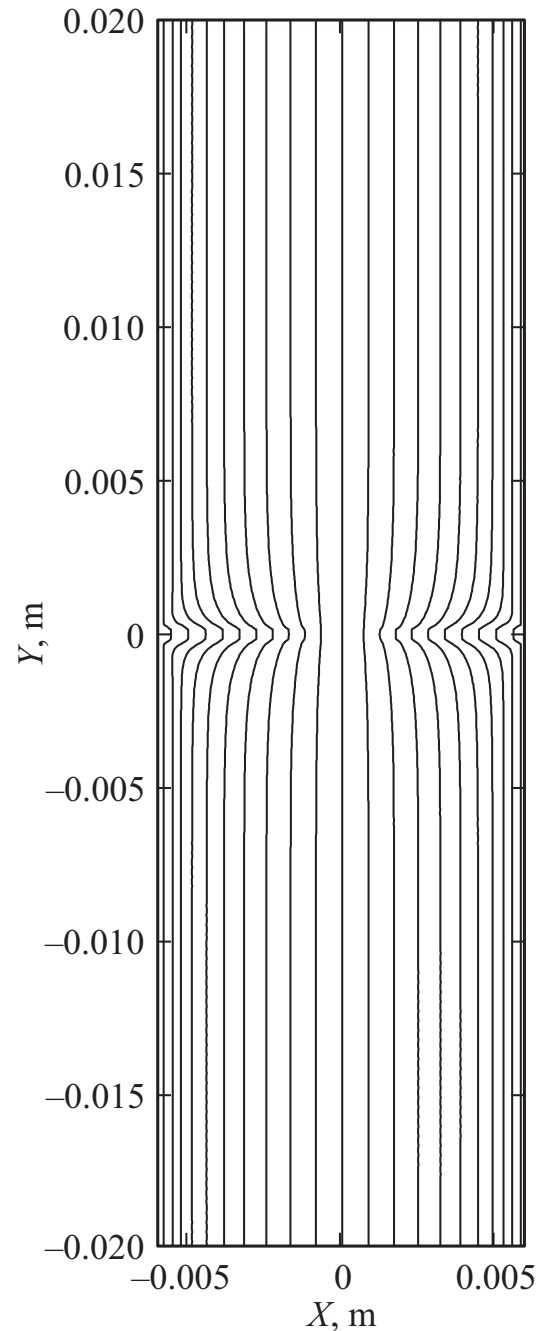

Pис. 4. Линии токов в системе шунт-ВТСП-лента с поперечной трещиной в области $Y=0$ без предварительной радиационной обработки. Одна линия соответствует току 20 А.

Расчет критического транспортного тока в структуре шунт - ВТСП-лента с трещиной дает значение $I_{\mathrm{c}}=437 \mathrm{~A}-$ на $\sim 8 \%$ меньше, чем в исходной бездефектной ленте. Это падение происходит несмотря на то, что эффективная толщина сверхпроводящего слоя в двухслойной структуре почти везде превышает толщину однослойной ленты в 2 раза и совпадают эти толщины только на трещине (см. рис. 2). Такая странная на первый взгляд ситуация объясняется тем, что пояс более тонкой ВТСП-пленки при $Y=0$ является концентратором магнитного поля.

Вблизи трещины амплитуда магнитного поля резко возрастает, что приводит к уменьшению плотности критического тока, а полный транспортный ток через систему определяется именно областью шунта под трещиной - „тонким местом““ сверхпроводящей линии.

Эффект концентрации магнитного поля на трещине иллюстрируют и объясняют рис. 4 и 5 . На рис. 4 представлены расчетные линии токов в системе шунт-дефектная ВТСП-лента. Плотность тока на этом рисунке пропорциональна густоте токовых линий. На этом рисунке видно, что вблизи трещины сверхпроводящий ток имеет не только компоненту $j_{Y}$ (единственная ненулевая компонента тока в бездефектной ленте), но и компоненту $j_{X}$. Знак компонент тока $j_{X}$ после трещины $(Y>0)$ противоположен знаку компоненты тока $j_{X}$ до трещины $(Y>0)$, и эта локальная циркуляция тока существенно увеличивает амплитуду магнитного поля $H_{Z}(X, Y)$ над трещиной.

Область трещины находится в критическом состоянии, что видно на рис. 5, где изображены линии уровней нормальной компоненты магнитного поля. В силу симметрии ленты нормальная компонента магнитного поля антисимметрична в поперечном направлении $X$, то есть $H_{Z}(X, Y)=-H_{Z}(-X, Y)$ и $H_{Z}(0, Y)=0$, т.е. в центральной части рис. 5 , где отсутствуют линии уровней, отсутствует и магнитное поле - сверхпро-

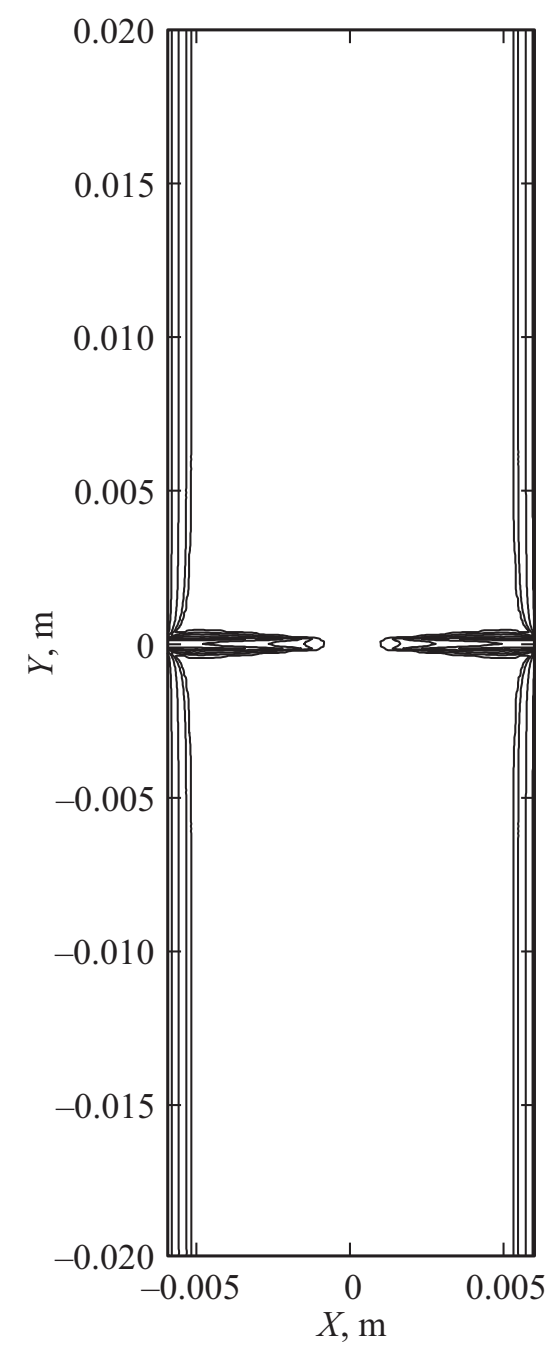

Рис. 5. Линии уровней амплитуды магнитного поля в системе шунт-ВТСП-лента с поперечной трещиной в области $Y=0$ без предварительной радиационной обработки. Расстояние между линиями равно $10000 \mathrm{~A} / \mathrm{m}$. 
водник находится в мейснеровском состоянии и полностью экранирует магнитное поле на поверхности структуры.

Магнитное поле отлично от нуля только на периферии ленты и в области трещины, где оно подавляет критический ток, протекающий через шунт. Ослабить эффект концентрации магнитного поля на краях трещины может предварительная (до соединения ленты и шунта) радиационная обработка краев трещины, приводящая к гладкому понижению плотности критического тока вблизи линии разлома сверхпроводника.

\section{3. Сверхпроводящие токи системы лента-шунт после радиационной обработки трещины ВТСП-ленты}

\section{1. Возможные режимы облучения}

Гладкое снижение плотности критического тока в дефектной ленте по мере приближения к трещине вдоль оси $Y$ может быть реализовано при облучении ленты через экранирующую маску переменной толщины. Толщина маски над трещиной равна нулю и возрастает при удалении от дефекта до толщины, достаточной для полного поглощения падающих быстрых частиц. Разрушение сверхпроводящего слоя возможно посредством облучения разного типа. Перспективы применения ВТСП-лент в ускорительной и космической технике стимулировали исследование деградации сверхпроводников в радиационных полях различного типа - при облучении ионами различных элементов, нейтронами, электронами и $\gamma$-квантами (смотри, например, работы $[1-3,13,19-21])$. Однако, среди многочисленных работ по данной тематике мы не обнаружили данные по необходимым нам режимам облучения. Для предварительной оценки режима работы ускорителя заряженных частиц нас интересуют минимальные энергии ионов водорода, гелия и кислорода, которые обеспечивают максимальное повреждение в середине сверхпроводящего слоя ВТСП-ленты на основе $\mathrm{GdBa}_{2} \mathrm{Cu}_{3} \mathrm{O}_{7-x}$. Облучение при этом проводится со стороны защитного слоя, состоящего из меди $(20 \mu \mathrm{m})$ и серебра $(2 \mu \mathrm{m}$, см. рис. 1). Также необходимо определение количества радиационных вакансий, создаваемых в сверхпроводящем слое этими ионами. Решение данной задачи проводилось нами посредством расчетного пакета SRIM [7], который работал в режиме „Quick: Kinchen-Pease“. При подборе расчетных параметров, вводимых в этот пакет, среди прочих, требуется определение пороговой энергии смещения атомов (displacement threshold energy, $E_{d}$ ) в слое ВТСП. Авторы различных работ приводят различные значения этой величины. В экспериментальной работе [22] из данных по облучению $\mathrm{YBa}_{2} \mathrm{Cu}_{3} \mathrm{O}_{7-x}$ быстрыми электронами $(0.1-2.4 \mathrm{MeV})$ для кислорода и меди получены величины $E_{d}$, равные 10 и $15 \mathrm{eV}$
Число вакансий, создаваемых одной быстрой частицей в защитном слое $(\mathrm{Cu}, \mathrm{Ag})$, сверхпроводящем слое $(\mathrm{GdBaCuO})$ и в подложке ВТСП-ленты (Hastelloy) при облучении

\begin{tabular}{c|c|c|c}
\hline $\begin{array}{c}\text { Тип частицы } \\
\text { и ее энергия }\end{array}$ & $\begin{array}{c}\text { Защитный } \\
\text { слой } \mathrm{Cu}, \mathrm{Ag}\end{array}$ & $\begin{array}{c}\text { Сверхпроводник } \\
\mathrm{GdBaCuO}\end{array}$ & $\begin{array}{c}\text { Подложка } \\
\text { Hastelloy }\end{array}$ \\
\hline $\mathrm{H} 2.27 \mathrm{MeV}$ & 15.5 & 18 & 3.6 \\
$\mathrm{He} 8.9 \mathrm{MeV}$ & 115 & 179 & 11 \\
$\mathrm{O} 72.5 \mathrm{MeV}$ & 1057 & 2515 & 42
\end{tabular}

соответственно. В экспериментальной работе [21] из данных по облучению $\mathrm{YBa}_{2} \mathrm{Cu}_{3} \mathrm{O}_{7-x}$ быстрыми электронами $(20-120 \mathrm{keV})$ для кислорода в плоскостях $\mathrm{CuO}_{2}$ и в цепочках получены величины $E_{d}$, равные 8.4 и $2.8 \mathrm{eV}$ соответственно. В теоретической работе [23] в зависимости от положения атома кислорода и направления его движения величина $\mathrm{Ed}$ изменяется в интервале 5-20 eV. В работе [24] для расчета в пакете SRIM были приняты значения $E_{d}$ для кислорода $-20 \mathrm{eV}$, для прочих атомов - $25 \mathrm{eV}$. Наиболее адекватными значениями нам представляются экспериментальные данные работы [21] 8.4 и $2.8 \mathrm{eV}$ для атомов кислорода в плоскостях и кислородных цепочках, соответственно. Величины энергий смещений прочих атомов выбраны по умолчанию, предлагаемыми вычислительным пакетом $(25 \mathrm{eV})$. В таблице приведены энергии быстрых частиц и количество вакансий, создаваемых одной частицей в ВТСП-слое и прилежащих к нему слоях. Из данных таблицы следует, что полное разупорядочение кристаллической структуры ВТСП-слоя толщиной $1.5 \mu \mathrm{m}$ произойдет при флюенсах $\sim 5 \cdot 10^{17}, 5 \cdot 10^{16}$ и $4 \cdot 10^{15} \mathrm{ion} / \mathrm{cm}^{2}$ для облучения ВТСП-ленты ионами водорода, гелия и кислорода соответственно. Самая высокая однородность плотности распределения радиационных вакансий по толщине ВТСП-слоя наблюдается при облучении ленты ионами водорода.

Отклонения плотности вакансий от среднего значения этой величины в сверхпроводнике равны $\sim 5,14$ и $12 \%$ при облучении ионами водорода, гелия и кислорода, соответственно. Лучшую однородности можно ожидать при облучении частицами с различными энергиями, или при облучении через различные маски.

\section{2. Постановка задачи расчета сверхпроводящих токов}

Резкое изменение направления вектора плотности тока при переходе через трещину, которое определяет величину магнитного поля над трещиной может быть устранено предварительной радиационной обработкой краев трещины до соединения дефектной ВТСП-ленты и шунта. Радиационные дефекты подавляют сверхпроводимость и обработка заключается в неоднородном 
облучении области трещины таким образом, что плотность критического тока гладко стремится к нулю на еe границах (при $Y=0)$. Для иллюстрации эффекта уменьшения величины магнитного поля над трещиной выбираем зависимость $h(Y)$ в выражении (1) в следующем виде:

$$
h(Y)=\left\{\begin{array}{ll}
1.5-0.5 \cdot \cos (\pi Y / a) ; & |Y| \leq a \\
2 ; & |Y| \geq a
\end{array} .\right.
$$

Зависимость (6) представлена на рис. 2 пунктирной линией.

\section{3. Результаты и обсуждение}

Расчет критического транспортного тока системы шунт - ВТСП-лента с трещиной после радиационной обработки дает величину $I_{\mathrm{c}}=467 \mathrm{~A}$, что ближе к току бездефектной ленты $I_{\mathrm{c}}=473 \mathrm{~A}$, чем к току системы шунт-лента без радиационной обработки $\left(I_{\mathrm{c}}=437 \mathrm{~A}\right)$.

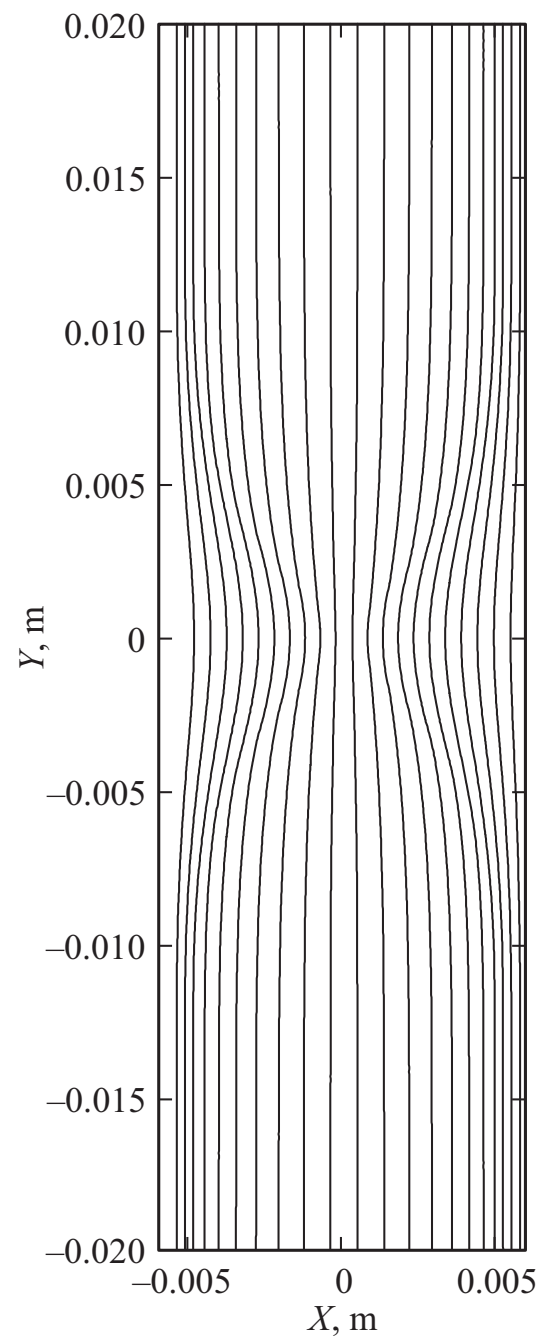

Pис. 6. Линии токов в системе шунт-ВТСП-лента с поперечной трещиной в области $Y=0$ после предварительной радиационной обработки. Одна линия соответствует току $20 \mathrm{~A}$.

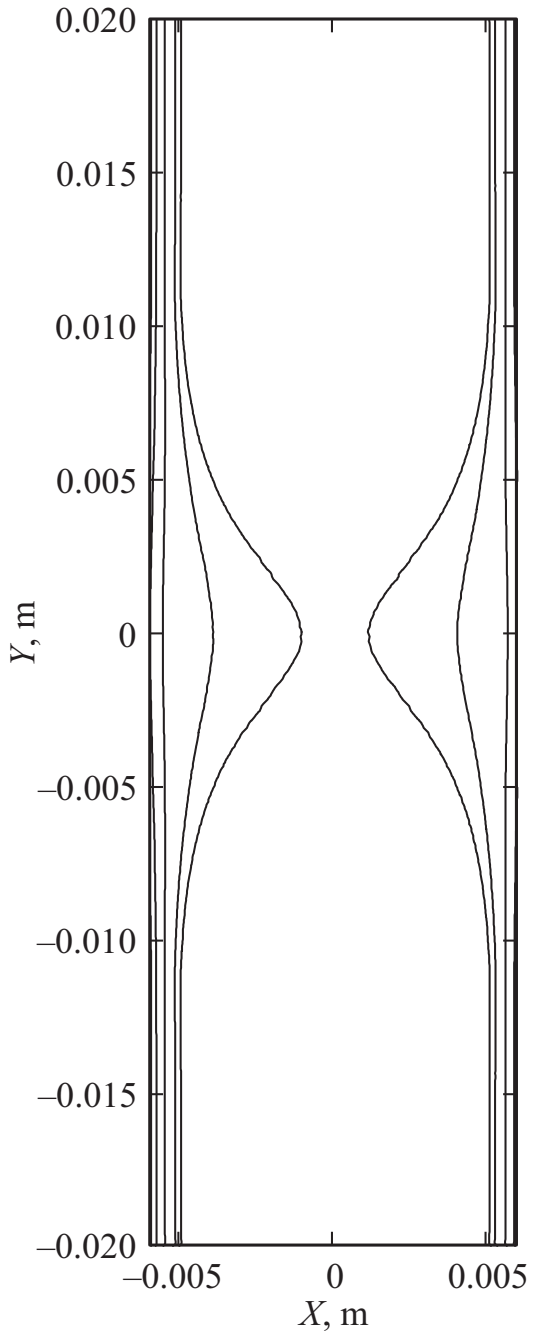

Рис. 7. Линии уровней амплитуды магнитного поля в системе шунт-ВТС-лента с поперечной трещиной в области $Y=0$ после предварительной радиационной обработки. Расстояние между линиями равно $10000 \mathrm{~A} / \mathrm{m}$.

На рис. 6 представлены линии токов в радиационнообработанной системе. На этом рисунке видно отсутствие излома направления тока вблизи трещины. На рис. 7 представлены линии уровней амплитуды магнитного поля $H_{Z}(X, Y)$. На этом рисунке видна делокализация магнитного поля в области трещины по сравнению с необлученным образцом (см. рис. 5). На рис. 8 сопоставлены амплитуды магнитного поля $H_{Z}(X, Y)$ при $X=-3 \mathrm{~mm},-20<Y<20 \mathrm{~mm}$ для бездефектной ленты и для бездефектной ленты, для системы шунт-лента без радиационной обработки и с обработкой. На этом рисунке видно, что пиковая амплитуда магнитного поля вблизи трещины более чем в 4 раза превышает аналогичную величину в бездефектной ленте, а пиковая амплитуда поля в обработанной системе превышает ее только на $\sim 15 \%$, чем и объясняется слабая деградация транспортного тока в обработанной системе. 


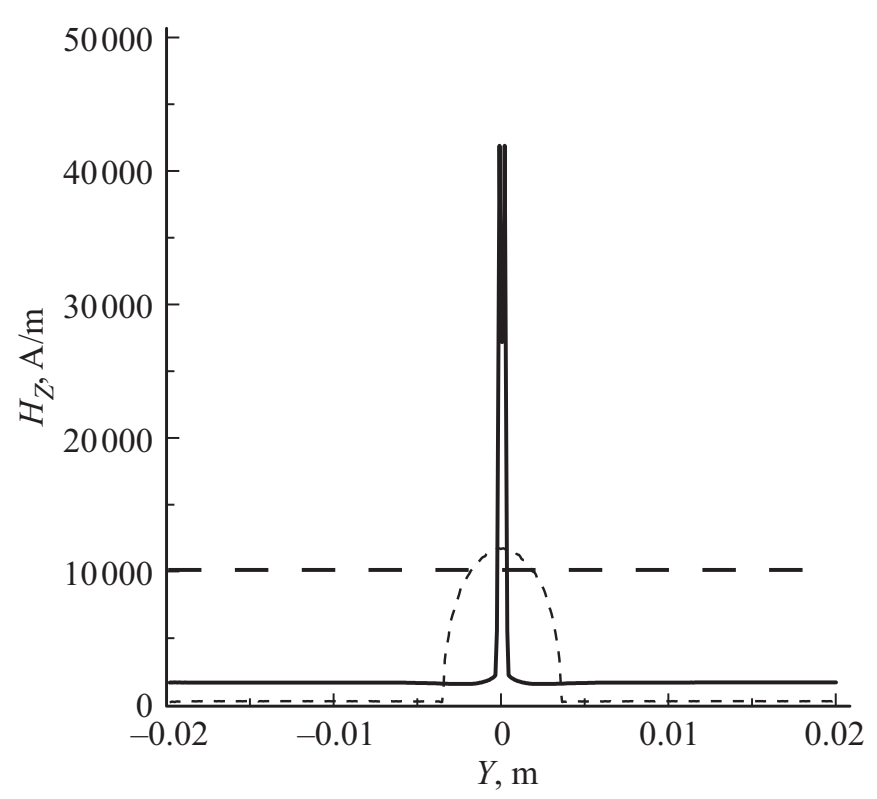

Рис. 8. Зависимость нормальной компоненты магнитного поля $H_{Z}(X=-3 \mathrm{~mm}, Y)$ в бездефектной ВТСП-ленте (жирная пунктирная линия), в системе шунт-ВТСП-лента с трещиной без радиационной обработки (жирная сплошная линия) и после радиационной обработки (тонкая пунктирная линия).

\section{4. Заключение}

Показано, что простое шунтирование участка ВТСПленты с трещиной не позволяет полностью восстановить полный транспортный ток через систему ВТСП-лента-шунт. Для полного восстановления транспортных характеристик системы необходимо управляемое снижение плотности критического тока в поврежденной ленте вблизи трещины посредством радиационной обработки. Представленные в настоящей работе результаты моделирования позволяют сформулировать рекомендации по восстановлению токонесущей способности ВТСП-ленты с поперечной трещиной. Ширина области трещины, которую необходимо подвергать неоднородному облучению через маску переменной толщины должна быть больше $24 \mathrm{~mm}$. Наиболее подходящим для этой цели является облучение ионами водорода.

\section{Финансирование работы}

Исследование выполнено при финансовой поддержке РФФИ и Госкорпорации „Росатом““ в рамках научного проекта № 20-21-00085.

\section{Конфликт интересов}

Авторы заявляют, что у них нет конфликта интересов.

\section{Список литературы}

[1] N. Habercorn, S. Suárez, Jae-Hun Lee, S.H. Moon, Hunju Lee. Solid State Commun. 289, 51 (2019).

[2] D.X. Fisher, R. Procopes, J. Emhofer, M. Eisterer. Supercond. Sci. Technol. 31, 044006 (2018).

[3] А.В. Троицкий, Т.Е. Демихов, Л.Х. Антонова, С.А. Кузьмичев, В.А. Скуратов, В.К. Семина, Г.Н. Михайлова. ФММ 120, 143 (2019).

[4] В.В. Деревянко, Т.В. Сухарева, В.А. Финкель. ФТТ 60, 465 (2018).

[5] I. Rudnev, A. Mareeva, N. Mineev, S. Pokrovskiy, A. Sotnikova. J. Phys.: Conf. Ser. 507, 0220 (2014).

[6] Д.В. Сотников. Исследование токонесущих свойств перспективных высокотемпературных сверхпроводящих материалов для электротехнических устройств. Дис. канд. тех. наук. ОАО ВНИИКП. Москва (2016). 126 с. https://www.vniikp.ru/media/documents/Dissertation_Sotnikov_ DV.pdf

[7] P. Biersack, L.G. Haggmark. Nucl. Instrum. Meth. Phys. Res. Sect. B 74, 257 (1980). WWW.srim.org.

[8] П.И. Безотосный, С.Ю. Гаврилкин, К.А. Дмитриева, А.Н. Лыков, А.Ю. Цветков. ФТТ 61, 2, 234 (2019).

[9] А.Н. Максимова, В.А. Кашурников, А.Н. Мороз, И.А. Руднев. ФТТ 63, 1, 65 (2021).

[10] А.И. Подливаев, И.А. Руднев. ФТТ 63, 6, 712 (2021).

[11] А.И. Подливаев, И.А. Руднев. ФТТ 63, 10, 1514 (2021).

[12] А.И. Подливаев, И.А. Руднев. ФТТ 64, 2, 161 (2022).

[13] Л.Х. Антонова, А.В. Троицкий, Г.Н. Михайлова, Т.Е. Демихов, С.В. Самойленков, А.А. Молодык, J. Noudem, P. Bernstein. Кр. сообщения по физике 44, 16 (2017). DOI: $10.3103 / \mathrm{S} 1068335617030034$

[14] A.I. Podlivaev, I.A. Rudnev, N.P. Shabanova. Bull. Lebedev Phys. Institute 41, 351 (2014).

[15] Th. Schuster, H. Kuhn, E.H. Brandt, M.V. Indenbom, M. Kläser, G. Müller-Vogt, H.U. Habermeier, H. Kronmüller, A. Forkl. Phys. Rev. B 52, 10375 (1995).

[16] G. Iannone, S. Farinon, G. De Marzi, P. Fabricattore, U. Gambardella. IEEE Trans. Appl. Supercond. 25, 8200107 (2015).

[17] A.I. Podlivaev, I.F. Rudnev. Supercond. Sci. Technol. 30, 035021 (2017). doi.org/10.1088/1361-6668/aa55aa

[18] I.A. Rudnev, A.I. Podlivaev. IEEE Trans. Appl. Supercond. 26, 8200104 (2016).

[19] F. Li, S.S. Wang, P. Zhao, S. Muhammad, X.Y. Le, Z.S. Xiao, L.X. Jiang, X.D. Ou, X.P. Ouang. Phys. Scr. 94, 105820 (2019).

[20] V. Bartůněk, J.L. Pérez-Diaz, T. Hlásek, L. Viererbl, H.A. Vratislavská. Ceram. Int. 46, 15400 (2020).

[21] S.K. Tolpygo, J.-Y. Lin, M. Gurvitch, S.Y. Hou, J.M. Phillips. Phys. Rev. B 53, 12462 (1996).

[22] A. Legris, F. Rullier-Albenque, E. Radeva, P. Lejay. J. Phys. France 3, 1605 (1993).

[23] Н.Н. Дегтяренко, В.Ф. Елесин, В.Л. Мельников. ЖТФ 65, 78 (1995).

[24] A. Khobnya, M.E. Pek, G. Greaves, M. Danaie, G.D. Brittles, S.E. Donnelly, F. Schoofs, A. Reilly, P.D. Edmondson, S. Pedrazzini. arXiv:1810.10477v3 [cond-mat.mtrl-sci].

Редактор К.В. Емцев 